\title{
Nanostructured carbon-based membranes: nitrogen doping effects on reverse osmosis performance
}

\author{
Josue Ortiz-Medina $^{1}$, Hiroki Kitano ${ }^{1,2}$, Aaron Morelos-Gomez ${ }^{1}$, Zhipeng Wang ${ }^{3}$, Takumi Araki ${ }^{1,4}$, \\ Cheon-Soo Kang ${ }^{3}$, Takuya Hayashi ${ }^{1,3}$, Kenji Takeuchi ${ }^{1,3}$, Takeyuki Kawaguchi ${ }^{3}$, Akihiko Tanioka $^{3}$, \\ Rodolfo Cruz-Silva ${ }^{1}$, Mauricio Terrones ${ }^{3,5}$ and Morinobu Endo ${ }^{1,3}$
}

\begin{abstract}
Ultrathin, flexible and highly water-permeable nanostructured carbon (NC)-based membranes are formed on porous polymer supports by plasma high-power impulse magnetron sputtering in order to fabricate carbon-based membranes for water desalination. The carbon membranes are produced at room temperature using mixtures of argon $(\mathrm{Ar})$, nitrogen $\left(\mathrm{N}_{2}\right)$ and methane $\left(\mathrm{CH}_{4}\right)$ as precursors, and this procedure constitutes a simple solvent-free, waste-free scalable process. Structural characterization, molecular simulation, water permeation and salt rejection assessments are used to correlate the performance and membrane structure. Molecular simulations indicate that nitrogen doping on the carbon-based membranes drastically modifies the pore distribution and avoids the formation of clustered regions of high-density carbons. The optimum NC-based membrane has up to $96 \%$ salt rejection rate for $0.2 \mathrm{wt} \% \mathrm{NaCl}$ saline water, with high water permeability ca.

$25 \mathrm{I} \mathrm{m}^{-2} \mathrm{~h}^{-1} \mathrm{MPa}^{-1}$. The NC-based membranes as active layers for desalination membranes exhibit attractive characteristics which render them a potential alternative to current polymeric technology used in reverse osmosis processes.
\end{abstract}

NPG Asia Materials (2016) 8, e258; doi:10.1038/am.2016.27; published online 1 April 2016

\section{INTRODUCTION}

Carbon thin films fabricated by a variety of methods are nowadays used in many applications, due to their attractive mechanical properties, chemical inertness and optical transparency, and are recurrently produced at an industrial scale. ${ }^{1}$ The possibility of nanostructure control of amorphous carbon (a-C)-based coatings, altering their properties such as density and composition by introducing $s p^{3}$ hybridized carbon atoms, heteroatoms such as hydrogen, ${ }^{2}$ silicon, ${ }^{3}$ fluorine, ${ }^{4}$ oxygen ${ }^{5}$ or nitrogen ${ }^{6}$ for example, in a similar way as doping of other carbon materials, allows us to expand a-C thin films range of applications. For example, taking advantage of its chemical inertness and non-toxicity, flexible hydrogenated a-C (or a-C:H) thin layers are used as gas barriers in packaging, where their barrier properties to $\mathrm{O}_{2}$ and $\mathrm{CO}_{2}$ are exploited for coating food and beverage containers. ${ }^{7,8}$ Furthermore, thin hard membranes of diamond-like-carbon, which is an a-C form with high proportion of $s p^{3}$ hybridized carbon, have been recently reported as the active layer of membranes used for organic solvent separation, ${ }^{9}$ exhibiting unique advantages of using such carbon thin membranes for the selective separation of gaseous or liquid species.

Generally, the basis of the attractive properties of a-C thin films is attributed to the special combination of $s p^{3}$ and $s p^{2}$ carbon hybridizations, but in fact, the formation of different chemical functionalities and hybridizations, especially for doped a-C formations, must be considered as key factors for carbon-based deposited films. Some commonly used techniques for a-C synthesis are magnetron sputtering, ${ }^{10}$ ion beam-assisted deposition, ${ }^{11}$ laser ablation, ${ }^{12}$ filtered cathodic vacuum $\operatorname{arc}^{1}$ and plasma-enhanced chemical vapor deposition, ${ }^{13}$ each one producing carbon structures with specific characteristics related to the physical process involved. Among these methods, high-power impulse magnetron sputtering (HiPIMS) has gained considerable interest, given the highly reactive nature of the sputtered material, which for carbon films is translated into increased $s p^{3}$ hybridized carbon capabilities ${ }^{14}$ and chemical modification possibilities.

In this work, we used HiPIMS to produce carbon-based membranes directly on porous polymeric substrates with the aid of an easily removable and non-toxic sacrificial layer, in order to construct a new type of flexible and robust membranes for water treatment. This practical method for preparing conventional porous substrates is explained, followed by details of nanostructured carbon (NC)-based membranes synthesis, chemical and structural characterization by means of electron and atomic force microscopies, electron energy loss, $\mathrm{X}$-ray photoelectron, IR and Raman spectroscopies. Water permeation

${ }^{1}$ Global Aqua Innovation Center, Shinshu University, Nagano, Japan; ${ }^{2}$ Kitagawa Industries Co., Kasugai City, Aichi, Japan; ${ }^{3}$ Institute of Carbon Science and Technology, Faculty of Engineering, Shinshu University, Nagano, Japan; ${ }^{4}$ Division of Computational Science and Technology, Research Organization for Information Science and Technology, Tokyo, Japan and ${ }^{5}$ Department of Physics, Department of Chemistry, Department of Materials Science and Engineering, \& Center for 2-Dimensional and Layered Materials. The Pennsylvania State University, University Park, PA, USA

Correspondence: Professor M Endo, Global Aqua Innovation Center, Institute of Carbon Science and Technology, Faculty of Engineering, Shinshu University, 4-17-1 Wakasato, Nagano 380-8553, Japan.

E-mail: endo@endomoribu.shinshu-u.ac.jp

Received 17 October 2015; revised 12 January 2016; accepted 31 January 2016 
and salt rejection efficiency were evaluated using a typical cross-flow setup for membrane tests, along with a simple assessment of membrane resistance to chlorine ions, exhibiting a clear enhanced performance when compared with previous works on carbon-based reverse osmosis (RO) membranes. ${ }^{15-17}$ Results from the characterization techniques were used to construct several molecular models of the possible carbon membrane microstructure, followed by molecular dynamics simulations used to understand the effect of nitrogen inclusion within the membrane nanostructure. We believe that the good performance of our NC-based membranes, along with the feasibility and scalability toward industrial scale, will contribute to develop optimized technologies for the upcoming high demands of clean water.

\section{EXPERIMENTAL PROCEDURES}

\section{Synthesis of NC-based membranes}

Figure 1 summarizes the process for NC-based membranes fabrication, which can be divided in two phases. First, deposition of a sacrificial layer of polyvinylpyrrolidone (PVP) solution on porous polysulfone (PSU) membrane (Alfa Laval-GR40PP ultrafiltration membrane), with a subsequent deposition of sputtered carbon layer by HiPIMS as the second phase. The PVP solution was prepared by dissolving PVP powder (Polyvinylpyrrolidone K30, Wako Pure Chemicals, Osaka, Japan) at $10 \mathrm{wt} \%$ in ethanol:water mixture (volume ratio 8:2). PVP solution was cast onto the PSU membrane by bar coating (glass bar of about $5 \mathrm{~mm}$ diameter), manually pouring PVP solution in excess and spreading it over PSU substrates (about $5 \times 5 \mathrm{~cm}$ ) attached with adhesive tape to a glass piece, and dried at room temperature for at least $10 \mathrm{~h}$. After the PVP coating process, the PSU membranes were used as substrates for NC-based membrane deposition by HiPIMS. Once the carbon layers are deposited on the substrates, the samples are immersed in water:ethanol mixtures (1:1) for $1 \mathrm{~h}$ in order to remove the sacrificial layer.

The NC-based membranes were produced by a multimodal thin film deposition system (ICF-500SHp, Nanotech, Chiba, Japan), operated in HiPIMS mode applying unipolar power pulses of $180 \mu$ s at $1.5 \mathrm{KHz}$, with a duty cycle $\sim 25 \%$ (see Supplementary Figure S1, data acquired using Agilent Technologies InfiniVision DSO-X 2012A oscilloscope). Four different sputter gas mixtures were used, with $\mathrm{Ar}, \mathrm{CH}_{4}$ and $\mathrm{N}_{2}$ in different proportions listed in Supplementary Table ST1, along with their corresponding process parameters, including target power density, average target power density values, deposition pressures and times. The sputtered carbon source was a solid graphite target (99.999\%, Myojo Kinzoku, Osaka, Japan), which was placed $142 \mathrm{~mm}$ away from the substrate surface. The deposition times for all cases were varied to obtain carbon-based membranes of $30 \pm 2$-nm thick (some membranes were produced with thicknesses of $25 \pm 2 \mathrm{~nm}$ for comparative purposes). During the deposition process, the plasma optical spectra was acquired via optic fiber using a portable spectrometer (Ocean Optics USB2000+ spectrometer), for each of the sputter gas mixtures. The acquired spectra can be observed in Supplementary Figure S2.

\section{Structural characterization of NC-based membranes}

NC-based membranes morphology was analyzed by scanning electron microscopy (SEM, Hitachi SU8000 FE-SEM) and atomic force microscopy (Agilent Technologies AFM 5500, Santa Clara, CA, USA) directly as produced on the porous PSU membranes. Transmission electron microscopy (TEM, JEOL JEM 2100F HR-TEM, Tokyo, Japan) was performed on carbon depositions sputtered on $\mathrm{KBr}$ crystals which were dissolved in deionized water for transferring free-standing pure carbon membrane onto TEM grids. Surface chemical analysis was carried out using an X-ray photoelectron system (XPS, Kratos Axis-Ultra, Manchester, UK) with Al Ko line and pass energy for the detector set to 160 and $20 \mathrm{eV}$ for low and high resolution scans, and a Raman spectrometer (Renishaw In-Via microscope, Gloucestershire, UK, $532 \mathrm{~nm}$ excitation laser) on carbon films sputtered on intrinsic Si (100) wafers. Attenuated total reflectance-Fourier transform infrared spectroscopy (Thermo Scientific Nicolet 6700 FT-IR, Waltham, MA, USA) was also carried out for carbon film samples sputtered on $\mathrm{KBr}$ crystals. Electron energy loss spectroscopy (EELS) was done along with TEM microscopy, using the same samples. $\mathrm{X}$-ray reflectivity (Rigaku SmartLab X-ray diffractometer) characterizations for density determination were carried out on carbon membranes on Si wafers. Finally, surface energy determination using water and ethylene glycol contact angle (Krüss Drop Shape Analyzer DSA30, Hamburg, Germany) were performed on different carbon membranes sputtered on Si wafers.

\section{Water permeation and desalination tests}

Water permeation and salt rejection performance were evaluated by laboratory scale cross-flow filtration system, in which circular membranes with effective area of $2.27 \mathrm{~cm}^{2}$ were placed in a stainless steel unit cell (see Supplementary Figure S3, for a system scheme). The cross-flow tests were carried out using transmembrane pressures from 1.0 to $5.0 \mathrm{MPa}$, whereas maintaining feed water flux at $300 \mathrm{ml} \mathrm{min}^{-1}$, corresponding to a water velocity at the membrane surface of $0.27 \mathrm{~m} \mathrm{sec}^{-1}$. The tested saline water concentrations were 2000 and 35000 p.p.m. of $\mathrm{NaCl}$ (corresponding to 0.2 and $3.5 \mathrm{wt} \%$, respectively). For permeated water, flux $(J)$ and salt rejection measurements were carried out by measuring permeate volume per time unit, and by measuring permeate conductivity (Horiba Scientific LAQUAact ES-71 conductivity meter, Kyoto, Japan), respectively. The reported data was calculated as follows:

$$
J=\frac{\Delta V}{A \Delta t}
$$

where $J$ is expressed in $1 \mathrm{~m}^{-2} \mathrm{~h}^{-1}$ at a given pressure (in $\mathrm{MPa}$ ), $\Delta V$ is the

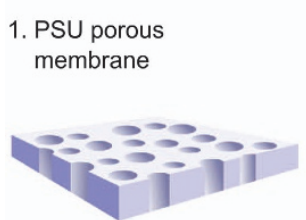

4. Carbon layer formation by sputtering/plasma

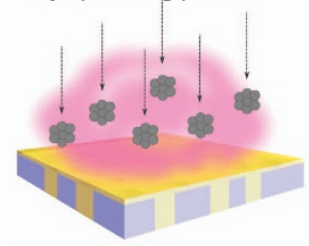

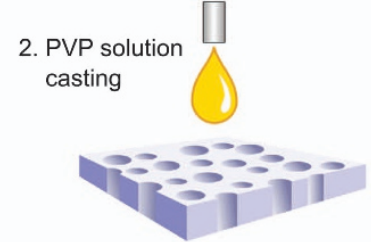

5. Sacrificial layer dissolving

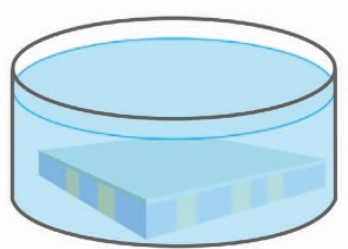

3. PVP sacrificial layer drying

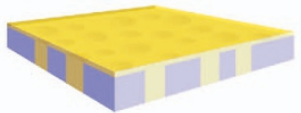

6. Pure carbon thin film on porous substrate membrane

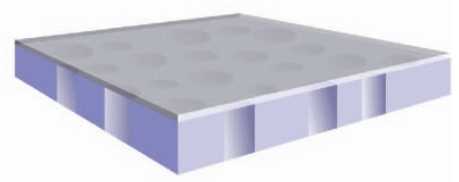

Figure 1 Steps for the NC-based membrane fabrication, which included the casting of PVP solution for sacrificial layer formation on porous PSU substrate, followed by sputtered/polymerized carbon deposition and sacrificial layer removal by ethanol:water mixture. 
permeated volume during time $(\Delta t)$, and $A$ corresponds to membrane effective area. For rejection performance:

$$
R=\left(1-\frac{C_{p}}{C_{f}}\right) 100 \%
$$

where, $R$ is the salt rejection, $C_{p}$ the permeate salt concentration, and $C$ corresponds to feed salt concentration. The reported data on permeate flux and salt rejection performance was the average of 3 to 5 independent readings, and the measurements were taken when flux and rejection performance stabilized over time (membranes tested during periods up to $24 \mathrm{~h}$ ). In addition, evaluations for all membranes were done after an initial compaction phase (at least for $1 \mathrm{~h}$ ) until a permeate flux reached a steady state.

\section{Chlorine resistance tests}

Chlorine resistance was evaluated by immersing the NC-based membranes in sodium hypochlorite aqueous solution (200 p.p.m.) solutions during $24 \mathrm{~h}$ at room temperature. After the chlorine treatment, the membranes were rinsed using deionized water and placed on cross-flow test cell for permeate flux and desalination performance measurements, which were carried out using two different saline concentrations: 0.2 and $3.5 \mathrm{wt} \% \mathrm{NaCl}$, at constant transmembrane pressures of 3.0 and $5.0 \mathrm{MPa}$ respectively. Additional chlorine resistance tests were carried out for membranes produced with $\mathrm{Ar}: \mathrm{N}_{2}: \mathrm{CH}_{4}$ mixture, by varying the nitrogen content from 48 to 18,15 and 9 vol\% approximately, while keeping $\mathrm{Ar}$ and $\mathrm{CH}_{4}$ content fixed.

\section{RESULTS AND DISCUSSION}

\section{Structural characterization}

The PSU membrane was used as a substrate for NC-based membrane depositions, and its morphology before carbon sputtering was observed by SEM (see Supplementary Figure S4). The typical PSU porous structure is clearly seen, from the uniform pore distribution and size from 10 to $70 \mathrm{~nm}$. After the PVP layer deposition, a uniform surface was observed, which was effective in providing a pore-free support for carbon film growth on top of the porous PSU substrate. It is important to highlight that PVP layer is non-toxic and easy to handle, making it a safe and environment-friendly option. An additional advantage of PVP as sacrificial layer is the enhanced adherence it provides for the carbon layer to PSU substrate, which has been recently reported to be observed to be strengthened under water conditions. ${ }^{18}$ A detailed discussion on the use of PVP as sacrificial layer can be found in experimental and Supplementary Section 3: PVP coating as sacrificial layer for carbon film formation.

Figure 2a shows SEM micrographs for NC-based membranes produced with different sputter gas mixtures, where top and side views are shown. From the side views, the carbon layer (average thickness of $31 \mathrm{~nm}$ ) could be distinguished from PSU substrate. It was also clear that the membranes exhibited a rippled surface, similar to diamond-like-carbon coatings on other flexible substrates like silicon-based rubbers, among others. ${ }^{19,20}$ However, it was also observed that the ripple morphology varied as a function of the gas mixture used during sputtering. The ripples were most likely related to the flexibility of the NC-based membrane, which could be explained by the presence of polymer-like-carbon interstitially produced between sputtered carbon clusters by plasma polymerization when $\mathrm{CH}_{4}$ is added to the gas mixture. It is noteworthy that pure polymer-likecarbon (which is also being explored by our group for developing carbon membranes) exhibited a very different nanostructuring when compared with sputtered carbon, which seems to be related to a different amount of functional groups and density. The $\mathrm{CH}_{4}$ effect can be contrasted by the atomic force microscopy analysis shown in Figure $2 b$, where topography and phase images for sputtered carbon layers deposited on $\mathrm{Si}$ are compared. It is clear from both topography and phase imaging that the use of $\mathrm{CH}_{4}$ drastically changed the surface morphology; when the carbon membranes were produced by $\mathrm{Ar}: \mathrm{CH}_{4}$ and Ar: $\mathrm{N}_{2}: \mathrm{CH}_{4}$, the sputtered membranes exhibited a rough surface with considerably big clusters, in contrast with a nearly flat surface when pure Ar was used (grain heights within $1 \mathrm{~nm}$ range). Interestingly, the incorporation of nitrogen seemed to help the coalescence of the cluster-like formations, resulting in height variations within ca. $8 \mathrm{~nm}$ with scattered nano-sized clusters when $\mathrm{Ar}: \mathrm{N}_{2}$ sputter gas was used.

TEM micrographs for NC-based membranes are shown in Figure 3a. The images were taken at the edges of carbon membrane samples, in order to look for thin sections which could allow a significant differentiation from samples produced by different sputter gas mixtures. The four types of carbon membranes exhibited an amorphous structure, because no clear plane orientation could be observed. EELS was performed in the areas shown by TEM, and the obtained spectra are shown in Figure 3b. EELS indicates that $\mathrm{CH}_{4}$ addition to the sputter mixture produced a significant increase in the $\sigma^{\star}$ signal, in contrast with the sputtered carbon membrane using only Ar. Moreover, the incorporation of $\mathrm{N}$ to the mixture sharpened the $\pi^{\star}$ region, which is associated with aromatic $s p^{2}$ carbons. An interesting effect could be identified in reference with a deep analysis on a-C:N films by Bhattacharyya et al., ${ }^{21}$ where the effect on nitrogen is identified as a downshift in energy for $\mathrm{N}$ concentrations $>10$ at $\%$, which was the case for the NC-based membranes analyzed here. Quantitatively, Figure $3 c$ shows the estimated $\left(s p^{3}\right)(100) /\left(s p^{2}+s p^{3}\right)$ ratios from EELS. The cited report ${ }^{21}$ also attributes the decrease in the ratio (from $c a .58 \%$ in Ar to $52 \%$ in $A r: N_{2}$ ), to the increase in $s p^{2}$ carbon bonding induced by a preferential $\mathrm{C}=\mathrm{N}$ bonds relative to $\mathrm{C}-\mathrm{N}$ bonds. Furthermore, the effect of introducing $\mathrm{CH}_{4}$ to the sputter mixture had a consistent effect of increasing the $\left(s p^{3}\right)(100) /\left(s p^{2}+s p^{3}\right)$ ratio, presumably because it promoted the formation of $\mathrm{C}-\mathrm{H}$ bonds.

Surface chemical analysis by XPS revealed features which could be related to the morphology observed by microscopy and ultimately with water permeation and salt rejection performance presented later. Figure 4 shows the XPS spectra for $\mathrm{C}, \mathrm{N}$ and $\mathrm{O}$ (1s) acquired for all the membrane types produced. Regarding to $\mathrm{C} 1 \mathrm{~s}$ results, three main binding energy regions were specified: $\mathrm{R}_{\mathrm{C} 1}, \mathrm{R}_{\mathrm{C} 2}$ and $\mathrm{R}_{\mathrm{C} 3} \cdot \mathrm{R}_{\mathrm{C} 1}$ corresponds to the region where typical graphitic $s p^{2}$ hybridized carbon appears, from 284 to $285 \mathrm{eV}$. The high-energy section of $\mathrm{R}_{\mathrm{C} 1}$ is also sensible to the contributions of $s p^{3}$ hybridized carbon with longer $\mathrm{C}-\mathrm{C}$ bonds, as has been described theoretically for a-C. ${ }^{22} \mathrm{R}_{\mathrm{C} 2}$ (from 285 to $287 \mathrm{eV}$ ) can be associated in this case to $s p^{2}$ carbon atoms bonded to $\mathrm{N}$ or $s p^{3}$ atoms, which is in accordance with the clear increase of peak intensity in this energy region for $\mathrm{N}$ containing NC-based membranes. $\mathrm{R}_{\mathrm{C} 3}$ region corresponds to $s p^{3}$ hybridized carbons with nitrogen to the nearest neighbors. For $\mathrm{N} 1 \mathrm{~s}$, the components were analyzed in terms of two energetic regions: $\mathrm{R}_{\mathrm{N} 1}$ and $\mathrm{R}_{\mathrm{N} 2}$, which according with previous investigations ${ }^{22,23}$ can be attributed to two coordinated $\mathrm{N}$, or three coordinated $\mathrm{N}$ bonded to saturated carbons $\left(\mathrm{R}_{\mathrm{N} 1}\right)$. The high-energy region $\mathrm{R}_{\mathrm{N} 2}$ would correspond to $\mathrm{N}$ sitting within $\mathrm{C}$ aromatic structures or having aromatic groups as first neighbors. It is noteworthy that the proportion of $\mathrm{N}$ in $\mathrm{R}_{\mathrm{N} 2}$ was higher for $\mathrm{Ar}: \mathrm{N}_{2}: \mathrm{CH}_{4}$ than the one for only Ar: $\mathrm{N}_{2}$ mixture (59 at $\%$ vs 50 at $\%$, from the area of deconvoluted peaks), which would suggest an increased presence of $\mathrm{N}$ groups normally found in polyamide membranes, and would also explain the salt rejection properties differences found for NC-based membranes produced, which will be described later. Finally, $\mathrm{O} 1 \mathrm{~s}$ spectra are also shown, splitting the species contributions in two energetic regions. $\mathrm{R}_{\mathrm{O} 1}$, which accounted for $\mathrm{O}$ bound to carbon atoms within 


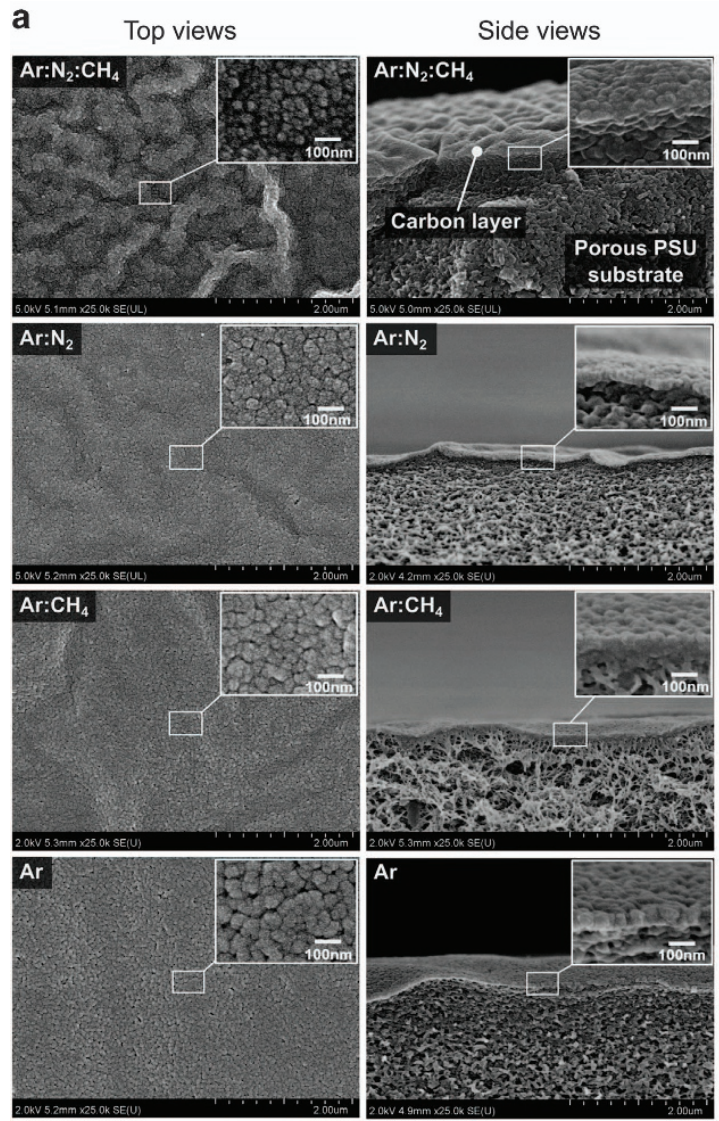

b Topography
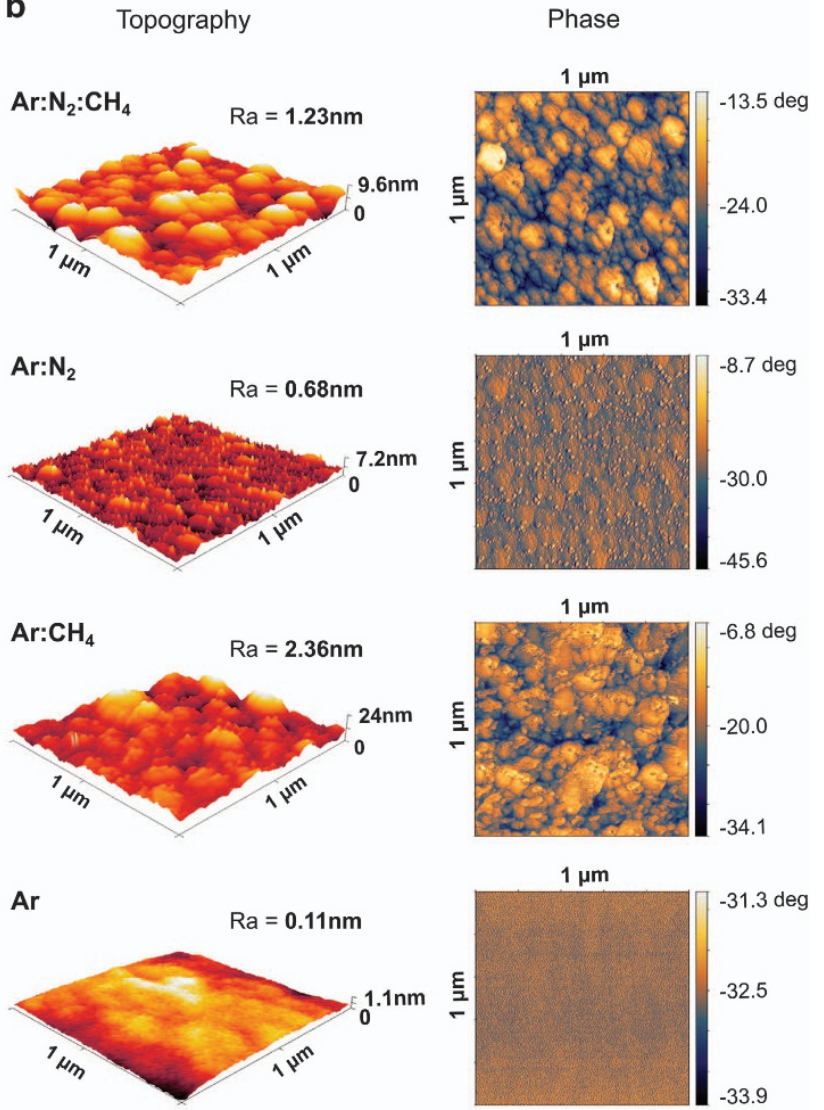

Figure 2 (a) SEM micrographs of sputtered NC-based membranes on PSU porous support, including membranes prepared by $\left(\mathrm{Ar}, \mathrm{N}_{2}\right.$ and $\left.\mathrm{CH}_{4}\right),\left(\mathrm{Ar}\right.$ and $\mathrm{N}_{2}$ ), ( $\mathrm{Ar}$ and $\mathrm{CH}_{4}$ ) and ( $\mathrm{Ar}$ ) as sputtering gas. (b) Atomic force microscopy analysis of carbon films deposited on $\mathrm{Si}$, by the different sputter gas mixtures. The topography and phase images are shown, along with the roughness value.

a

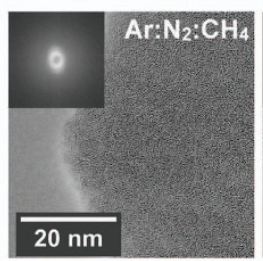

b

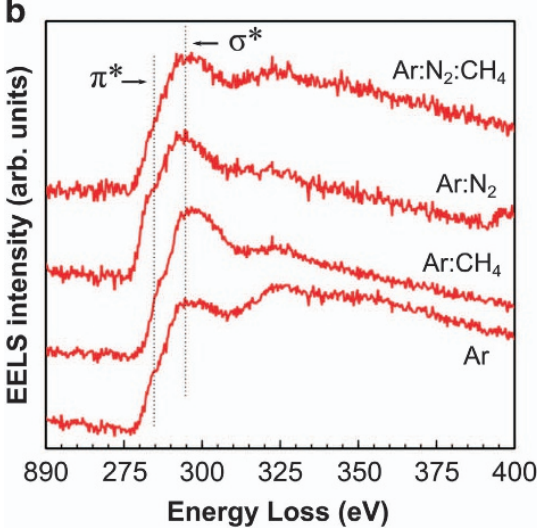

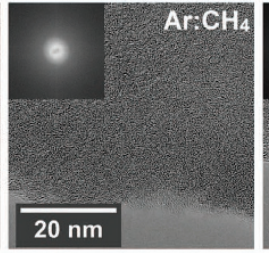
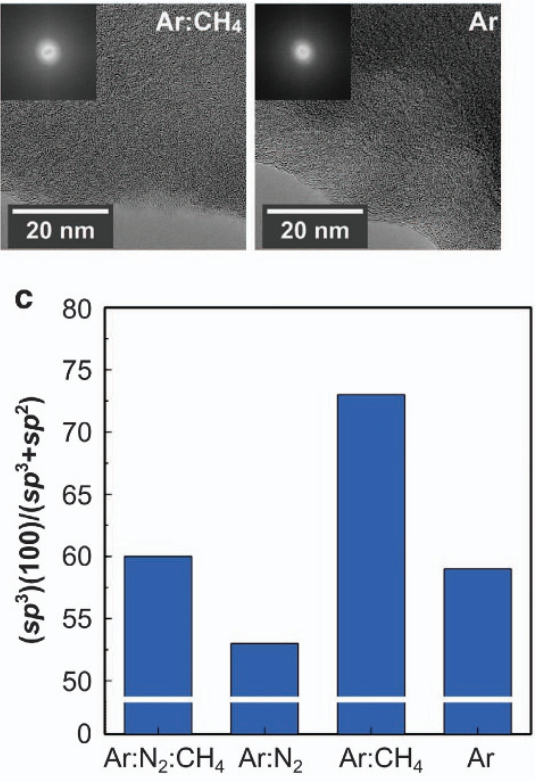

Figure 3 (a) TEM micrographs for the sputtered NC-based membrane samples using different gas mixtures. (b) EELS spectra in the carbon K-edge region, for the studied carbon membranes. The dotted vertical lines depict the energy loss for $1 \mathrm{~s}$ to $\pi^{*}$ and $1 \mathrm{~s}$ to $\sigma^{*}$ peaks. (c) Calculated $\left(s p^{3}\right)(100) /\left(s p^{2}+s p^{3}\right)$ ratios from EELS $\pi^{*}$ and $\sigma^{*}$ peaks. 

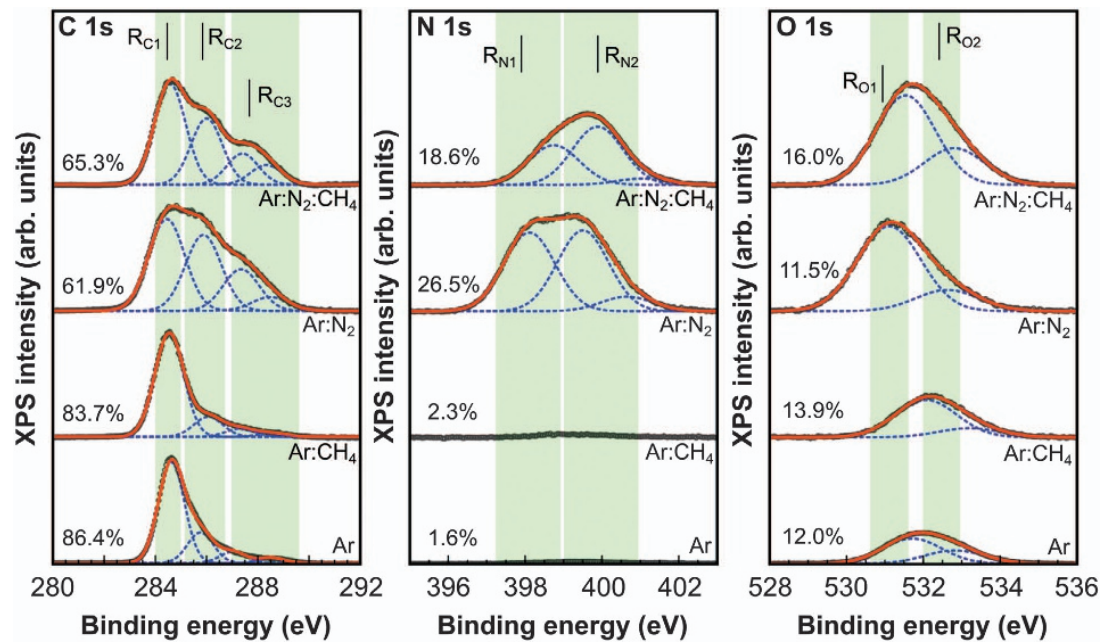

Figure 4 XPS spectra for sputtered NC-based membranes, produced with different gas mixtures. The $\mathrm{C} 1 \mathrm{~s}, \mathrm{~N} 1 \mathrm{~s}$ and $\mathrm{O}$ 1s peaks are shown, including their atomic percentage of total composition, and a deconvolution for general chemical species identification.
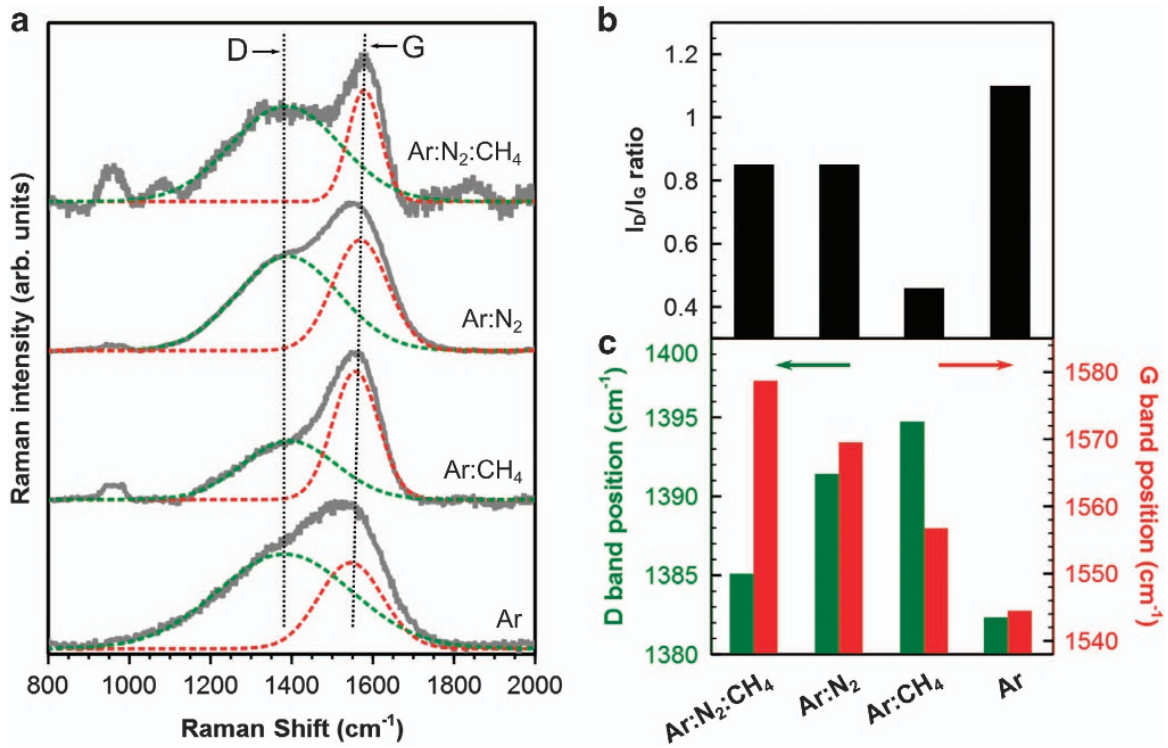

Figure 5 (a) Visible Raman spectra for NC-based membranes, with deconvoluted $D$ and $G$ bands, and changes in (b) $I_{D} / I_{G}$ ratios, (c) D and G band position shiftings.

nitrogenized groups, and $\mathrm{R}_{\mathrm{O} 2}$, where other typical carbon-oxygen groups were found ${ }^{24}$ (see Supplementary Figure S5 for the chemical models corresponding to each described XPS region). It is necessary to mention here that, given the fact that oxygen was not used as precursor for NC-based membranes synthesis, it was most likely reacted after the plasma process, due to the existence of very active surface radicals when samples were exposed to atmospheric conditions after synthesis.

Raman spectroscopy is widely used for carbon-based structures characterization, given the well-identified signatures that arise from graphitic $\left(s p^{2}\right)$ carbon networks. Figure 5a displays visible Raman spectra (532 nm excitation line) for the produced NC-based membranes, along with corresponding changes in $\mathrm{D}$ and $\mathrm{G}$ bands intensity ratios $\left(\mathrm{I}_{\mathrm{D}} / \mathrm{I}_{\mathrm{G}}\right)$ and shiftings (Figures $5 \mathrm{~b}$ and $\mathrm{c}$ ). Analysis of Raman revealed interesting changes in function of the used gas mixture for membrane deposition. According with Ferrari and Robertson, ${ }^{25,26}$ the introduction of compounds like $\mathrm{CH}_{4}$ to the mixture would explain the decrease of $\mathrm{I}_{\mathrm{D}} / \mathrm{I}_{\mathrm{G}}$ ratio, given the amorphization of nano-sized graphite clusters toward carbon chains, thus destroying the resonant origin of $\mathrm{D}$ band (namely, defects on $s p^{2}$ networks). On the other hand, the upshifting of the $\mathrm{G}$ band position could be attributed to an increasing amount of $s p^{3}$-like bonds, presumably produced by $\mathrm{C}-\mathrm{H}$ bondings along the chains. In contrast, nitrogen incorporation tended not to significantly alter the $\mathrm{D}$ band intensity nor its position, given that it appeared to preferentially locate into carbonaceous ring structures, which breathing mode is important source for Raman D mode. This is especially noticeable for the case of Ar: $\mathrm{N}_{2}: \mathrm{CH}_{4}$, considering that XPS revealed a slight preferential location of $\mathrm{N}$ atoms within aromatic carbon structures when compared against $\mathrm{N}$ atoms in $\mathrm{Ar}: \mathrm{N}_{2}$. 
Attenuated total reflectance-Fourier transform infrared spectroscopy analysis also revealed important differences in the presence of functional groups within the structure of NC-based membranes, as it is shown in Figure 6. Carbon films from pure $\mathrm{Ar}$ as sputter gas exhibited practically no functional groups, which was the expected result as only nano-sized graphitic clusters would be conforming the carbon layer. For the rest of the gaseous mixtures (Ar: $\mathrm{CH}_{4}, \mathrm{Ar}: \mathrm{N}_{2}$ and Ar: $\mathrm{N}_{2}: \mathrm{CH}_{4}$ ), functional moieties related with oxidation functionalities $\left(\mathrm{OH}\right.$ in the $3000-3500 \mathrm{~cm}^{-1}$ range) and saturated carbons (2800-3000 $\mathrm{cm}^{-1}$ ) could be observed, which seemed to form a considerable part of the $s p^{3}$ hybridized carbon found. C-N and C-O derivations (1500-1800 and 2100-2250 $\mathrm{cm}^{-1}$ ) were also detected. By comparing the recorded spectra, it was noticeable that the Ar: $\mathrm{N}_{2}: \mathrm{CH}_{4}$ sputter mixture produced the NC-based membranes with the strongest absorbance from $\mathrm{OH}$ functionalities, with $\mathrm{Ar}: \mathrm{CH}_{4}$ and Ar: $\mathrm{N}_{2}$ mixtures showing lower absorption and finally Ar with practically no $\mathrm{OH}$ groups. This trend is in accordance with XPS results

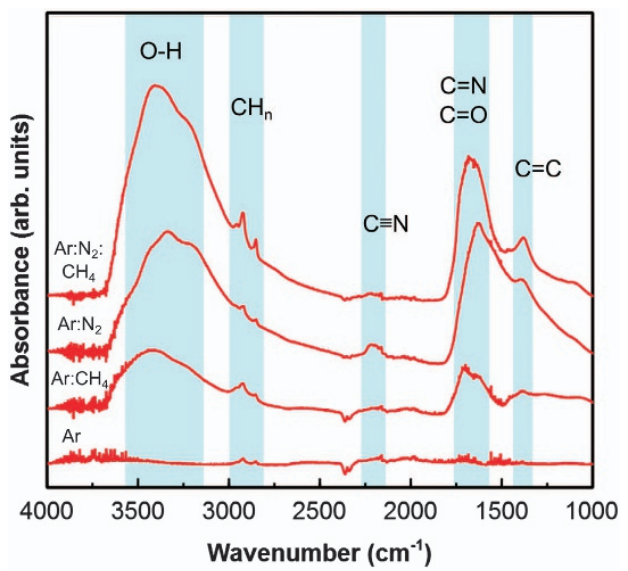

Figure 6 ATR-FT-IR spectra for NC-based film samples. The shadowed areas correspond to characteristic functional groups fingerprints, related with their different chemical composition and thus, with properties such as water permeability. described before, where the presence of oxygen functional groups could be explained by their enhanced adsorption from atmosphere when exposed to air, due to the highly reactive groups produced when using $\mathrm{N}_{2}$ and $\mathrm{CH}_{4}$ in sputter mixtures. The presence of such functionalities would play an important role in terms of water permeation in NC-based membranes, as will be observed later. Regarding to nitrogen containing functionalities, the spectra showed an increased absorption due to $\mathrm{C} \equiv \mathrm{N}$ and $\mathrm{C}=\mathrm{N}$ groups (around 2200-2300 and 1600-1750 $\mathrm{cm}^{-1}$, respectively), as could be expected from the introduction of $\mathrm{N}_{2}$ gas to the sputter mixture. It is noteworthy that for all the membrane types, there was a small absorption peak due to $\mathrm{C} \equiv \mathrm{N}$ groups, which was more pronounced when $A r: N_{2}$ mixture was used, suggesting that some $\mathrm{N}$ traces could have been released from PSU susbtrate or PVP during plasma process. Nevertheless, it is interesting to note that when $\mathrm{Ar}: \mathrm{N}_{2}: \mathrm{CH}_{4}$ mixture was used, the $\mathrm{C}=\mathrm{N}$ bonding seemed to predominate over the $\mathrm{C} \equiv \mathrm{N}$, which was in accordance with the XPS results, as well as an increase of $s p^{3}$ carbon when $\mathrm{CH}_{4}$ was used, presumably by the saturation of carbon chains as proposed from EELS and Raman results. Comparing these results with related previous works, ${ }^{27,28}$ it seems that the effect of introducing $\mathrm{CH}_{4}$ to the Ar: $\mathrm{N}_{2}$ mixture had a substantially different effect contrasted with only $\mathrm{H}_{2}$, for instance. The decrease in $\mathrm{C} \equiv \mathrm{N}$ groups when including $\mathrm{CH}_{4}$ in the NC-based membranes could be explained by the fact that dissociated $\mathrm{CH}_{4}$ molecules took a bigger share of the plasma kinetic energy from $\mathrm{N}_{2}$ ions, reducing the formation of energy demanding groups like $\mathrm{C} \equiv \mathrm{N}$.

Contact angle is a commonly used technique as a straightforward assessment of hydrophobicity/hydrophilicity properties for surfaces, and furthermore, it can be used for determination of key parameters such as surface energy. Figure 7 a shows the water droplet contact angle photographs and degrees for NC-based films deposited on Si wafers, and Figure $7 \mathrm{~b}$ shows the corresponding surface energy calculated from measurements made using water and ethylene glycol (see details for calculations in Supplementary Section 5: Surface energy calculations). Surface energy was represented as the sum of two components: polar and dispersive. The dispersive component is related with non-polar groups, which would contribute to the relative high hydrophobicity of carbon films sputtered using only Ar. Interestingly, the addition of a
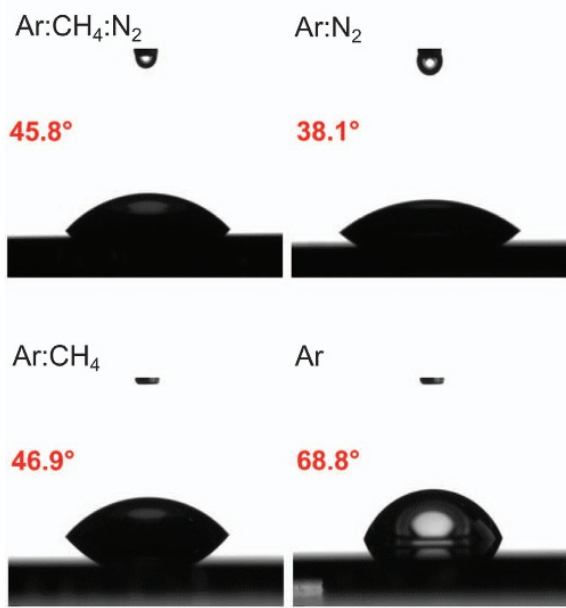

$\operatorname{Ar}$

$68.8^{\circ}$

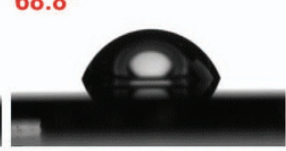

b

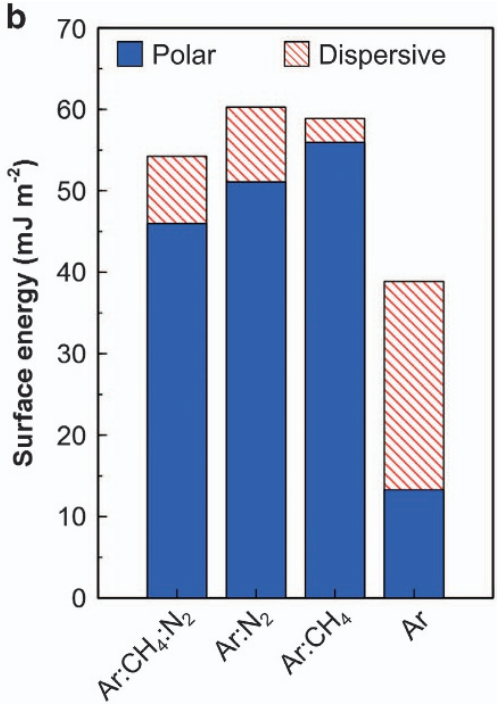

Figure 7 (a) Pure water contact angle photographs for the NC-based films deposited on Si wafers, with their corresponding angle value. (b) Surface energy scheme, where the total energy is shown with its polar and dispersive components for each type of carbon layer. 
either $\mathrm{CH}_{4}$ or $\mathrm{N}_{2}$ to the sputter mixture increased not only the overall surface energy, but specifically the polar component of it, effectively decreasing the water contact angle (more hydrophilic surface). The high-dispersive component for films made using Ar would suggests potential good performance for non-polar liquids separation, such as organic solvents like pentane, hexane, toluene, chloroform and so on, which has been already proposed experimentally for ultrathin diamond-like-carbon-based membranes. ${ }^{9}$

\section{Salt rejection properties of NC-based membranes}

For water permeation and salt rejection tests, the first step was to evaluate the four NC-based membranes varieties, by means of a cross-flow setup (Supplementary Figure S3). Supplementary Figure S6 shows the permeance and rejection performance for tests performed using $0.2 \mathrm{wt} \% \mathrm{NaCl}$ water. The $\mathrm{Ar}: \mathrm{N}_{2}: \mathrm{CH}_{4}$ membrane showed the best rejection and permeate flux among all experiments, with a desalination performance ca. $96 \%$ and permeance of $21.6 \mathrm{~m} \mathrm{~m}^{-2} \mathrm{~h}^{-1} \mathrm{MPa}^{-1}$. In terms of salt rejection, it is noteworthy that Ar: $\mathrm{N}_{2}$ membrane ran second place, with $71 \% \mathrm{NaCl}$ rejection. This suggests fundamental effects caused by nitrogen within the carbonbased structure of the film, and it is in accordance with the role that nitrogen groups would have in many modern aromatic polyamide RO membrane technologies. ${ }^{29}$ The carbon membranes prepared by $\mathrm{Ar}$ and $\mathrm{Ar}: \mathrm{CH}_{4}$ sputter mixtures performed poorly with regard to salt rejection. Nevertheless, the presence of $\mathrm{CH}_{4}$ was crucial for the highrejection and flux performance, as evidenced by $\mathrm{Ar}: \mathrm{N}_{2}: \mathrm{CH}_{4}$ membrane. After analysis of structural characterization data, the differences on rejection and permeate flux among NC-based membranes could be attributed to mainly two factors: (1) nitrogen containing groups, which resulted fundamental for good rejection performance specially in configurations where it was surrounded by $s p^{2}$-like carbon environment, and (2) the presence of $\mathrm{CH}_{4}$, which when combined with nitrogen in sputter mixture seemed to promote a preferential arrangement of $\mathrm{N}$ atoms among aromatic carbonaceous environments, while keeping an hydrophilic environment throughout the membrane structure which accounted for improved permeate flux. The salt rejection performance dependency on the presence of nitrogen containing groups is well-established for aromatic polyamide RO technology, where the charged amine moieties presumably exert a repulsive effect on the solvated ions, whereas keeping the polymeric network hydrophilic enough to allow the water diffusion throughout the polymer chains. The effects of $\mathrm{CH}_{4}$ were less clear and difficult to explain unequivocally. Apparently it would be related to the saturation of carbon aliphatic chains and forcing of nitrogen to incorporate in the proximities of or into aromatic structures. This hypothesis is supported by the spectroscopic results, given the preferential formation of pyridine-like groups and $\mathrm{N}$ atoms close to aromatic carbon rings as seen in XPS, and the increase of $s p^{3}$ hybridizations ratio when $\mathrm{CH}_{4}$ was used in the sputter mixture.

Given the obtained results for rejection and permeate flux performance, we focused on NC-based membranes produced by Ar: $\mathrm{N}_{2}: \mathrm{CH}_{4}$ mixture, by testing with 0.2 and $3.5 \mathrm{wt} \% \mathrm{NaCl}$ water solutions, and transmembrane pressures from 1.0 to $5.0 \mathrm{MPa}$. Figure $8 \mathrm{a}$ shows the salt rejection and permeate flux values for the evaluated membranes, exhibiting a typical RO transport behavior, with a stable water diffusion rate (parallel flux/transmembrane pressure slopes) independent of $\mathrm{NaCl}$ concentration. We also carried out permeate and rejection tests for thinner $\mathrm{Ar}: \mathrm{N}_{2}: \mathrm{CH}_{4}$ membranes, in order to compare the effect of thickness in RO performance. The results are shown in Supplementary Figure S7, where it can be observed that thickness had an important effect on performance, not only because its direct relationship with water flux, but also given that defects were easily to appear for thinner membranes. In addition, we assessed the effects of decreasing feed flux on the salt rejection performance, as it would be related with further concentration polarization evaluations. The results can be seen in Supplementary Figure S8, where a rejection degradation of around 7\% (using $3.5 \mathrm{wt} \%$ $\mathrm{NaCl}$ water) was observed for a reduced feed flux from 270 to $75 \mathrm{ml} \mathrm{min}^{-1}$.

At this point it is important to highlight that our NC-based membranes outperformed previous attempts to produce carbonbased membranes for RO applications, and also presented key advantages in terms of their processing and preparation. Supplementary Figure S9 presents a comparative scheme between RO membranes prepared by different groups, using plasma polymerization of organic precursors also directly on flexible substrates. ${ }^{15-17,30}$ As can be observed in the scheme, our NC-based membranes exhibited a high-rejection performance (>95\%), comparable with
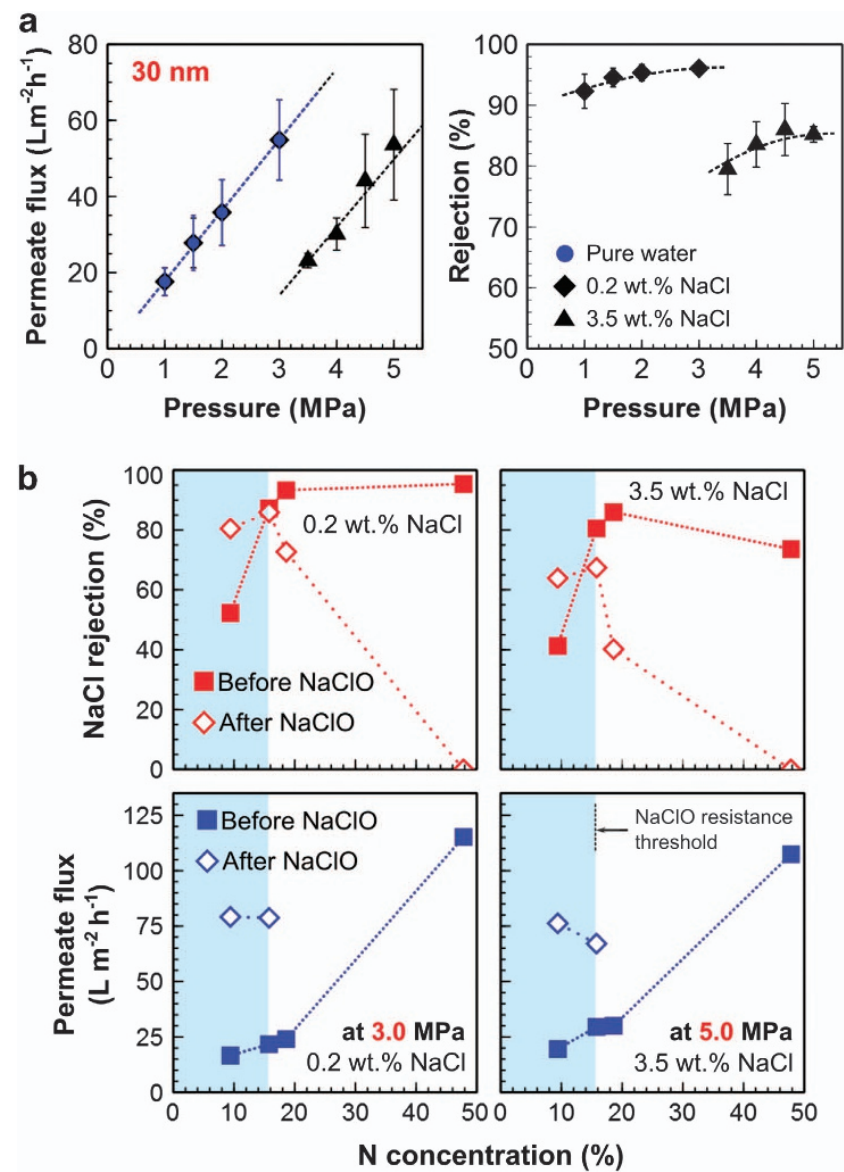

Figure 8 (a) Permeate flux and water desalination performance plots, for NC-based membranes deposited using $\operatorname{Ar}: \mathrm{N}_{2}: \mathrm{CH}_{4}$ (30 nm thickness). The results are shown for contrasting permeation for pure and concentrated saline water $(0.2$ and $3.5 \mathrm{wt} \% \mathrm{NaCl})$, and their corresponding salt rejection rates, in function of pressure under cross-flow tests. The segmented lines are used as an eye-guide. (b) Salt rejection performance degradation by $\mathrm{NaClO}$ exposure. The plots show the degradation as function of nitrogen concentration for tests made on $\mathrm{Ar}: \mathrm{N}_{2}: \mathrm{CH}_{4} \mathrm{NC}$-based membrane. The related permeate flux is also shown. Note that performance for tests on NC-based membranes with nitrogen concentrations above a threshold of $16 \mathrm{vol} \%$ (in sputter gas mixture) resulted in very high flux and reduced salt rejections, due to highly damaged carbon membrane. 


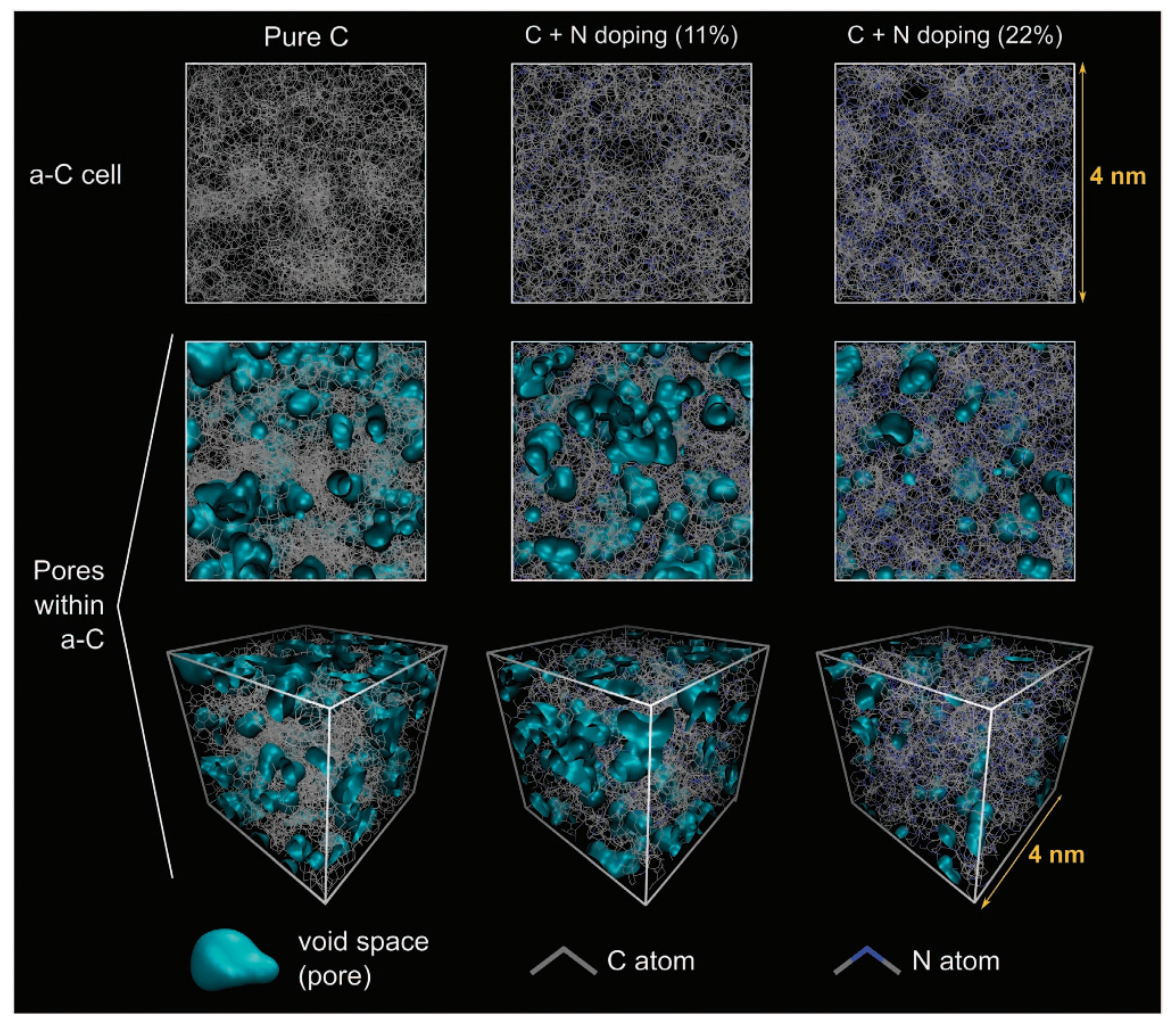

Figure 9 a-C cells constructed using Tersoff potential and simulated annealing. The cells are made of pure carbon atoms (represented as a gray sticks) and different concentrations of nitrogen ( 11 at\% and 22 at\%, represented as blue sticks). The middle and bottom cells show volumetric surfaces representing the pores within a-C structure, seen as cyan blobs.

the best values from Peric et al., ${ }^{17}$ Yasuda et al. ${ }^{16}$ and Hinman et al. ${ }^{15}$, but with a considerable higher permeate flux ( $c a .4 .5$ times higher), for $0.2 \mathrm{wt} \% \mathrm{NaCl}$ saline water. On the other hand, for the $3.5 \mathrm{wt} \% \mathrm{NaCl}$ water, the salt rejection fell to $c a$. $86 \%$, but kept permeate flux around four times higher when compared with the work by Yasuda et al. ${ }^{16}$ It is important to note here also that the tests for water permeation and salt rejection were carried out at much lower transmembrane pressures during cross-flow filtration: from 1.0 to 5.0 MPa, whereas previous carbon-based membranes were evaluated at $10.3 \mathrm{MPa}$. Thus, these NC-based membranes presented an advantage for applications, given that using membranes with high salt rejection at lower pressures would reduce the energy consumption during operation of desalination modules. Furthermore, the possibility of synthesizing uniform carbon-based films at nanoscale thickness directly on porous and flexible substrates is another advantage given by PVP as sacrificial layer for carbon deposition (see Supplementary Figure S4, along with extended details in Supplementary Section 3), which is also cost-effective and environment friendly.

\section{Chlorine resistance evaluation}

Membrane degradation by chlorine ions attack is among the most important challenges that current polymeric RO membranes face, given that the use of $\mathrm{NaClO}$ is a widespread method for killing bacteria and other microorganisms commonly present in contaminated water. In the case of NC-based membranes, our initial assessments on chlorine resistance resulted in heavily damaged membranes, especially for the ones with higher nitrogen content. From this point on, we assessed the effects of reducing nitrogen content from the original Ar: $\mathrm{N}_{2}: \mathrm{CH}_{4}$ sputter mixture on the salt rejection and water permeation characteristics of NC-based membranes. Figure $8 \mathrm{~b}$ shows the results for chlorine degradation tests for membranes based on NC-based films produced using four different nitrogen concentrations expressed as volume fraction in sputter gas mixtures. It was clear that the original $A r: \mathrm{N}_{2}: \mathrm{CH}_{4}$ membrane (that is, $\mathrm{N}$ concentration ca. $48 \mathrm{vol} \%$ ) was severely damaged after chlorine exposure, with salt rejection performance falling from 96 and $74 \%$ (for 0.2 and $3.5 \mathrm{wt} \%$ saline water respectively), to no salt rejection at all. Simultaneously, the permeate flux changed from $c a .115 \mathrm{~m}^{-2} \mathrm{~h}^{-1}$ at $3.0 \mathrm{MPa}$ to a practically unimpeded flux. Chlorine-induced degradation of NC-based membranes was considerably reduced for carbon films synthesized using ca. $16 \mathrm{vol} \%$ of nitrogen, where a threshold for keeping salt rejections of $86 \%$ for $0.2 \mathrm{wt} \%$ saline water, and $67 \%$ for $3.5 \mathrm{wt} \%$ saline water after chlorine exposure was obtained. It is noteworthy that for the NC-based membranes with low nitrogen concentrations (below $16 \mathrm{vol} \%$ ), an important increase in water permeation was observed after chlorine exposure, without degradation of the salt rejection properties, resulting in an increased performance.

\section{Theoretical simulation on a-C structure}

Three different a-C molecular models were constructed and analyzed in order to elucidate the changes that nitrogen induced within the a-C nanostructure, as a model of our NC-based membranes. The models were: (a) pure a-C, (b) a-C:N with 11 at $\%$ of $\mathrm{N}$ and (c) a-C:N with 22 at $\%$ of $\mathrm{N}$ concentration. Figure 9 depicts the models after molecular relaxation using molecular dynamics routines considering Tersoff potentials, plus simulated annealing simulations for structural modifications (details on the calculations can be found in Supplementary Section 6: Theoretical simulations details for DLC cell models and Supplementary Figure S10). Interstitial voids are 
represented with surfaces (cyan blobs) in order to visualize the pore density and channels formation as a function of nitrogen content. The free volume vs total cell volume ratio were calculated as $0.46,0.37$ and 0.30 , for pure $\mathrm{C}, \mathrm{C}+\mathrm{N}$ doping (11 at $\%)$ and $\mathrm{C}+\mathrm{N}$ doping (22 at $\%)$, respectively. By comparing these theoretical results with the experimental results such as water permeability and contact angle, it can be said that the void spaces within a-C structure were not the only factor directly correlated with water permeation capabilities, because the non-polar nature of pores within pure carbon structures could affect the charge particles passage or adsorption, ${ }^{31}$ and thus could hinder the passage of water molecules, as has been observed experimentally here and in other previous works, where pure carbon membranes exhibit low water flux when compared with non-polar organic molecules permeation. ${ }^{9,30}$ Despite the theoretical models analyzed were very simplified with respect to experimental structures of NC-based membranes, the simulation confirmed an experimental observation: the incorporation of nitrogen within the carbonaceous structure tended to decrease the clustering, thus resulting in a more homogeneous structure which was noticeable by the reduction on the interstitial voids. It is important to note also that, although the a-C molecular models were constructed using a fixed amount of atoms, the carbon clustering could explain the different density values from experimental density measurements on NC-based films (see Supplementary Section 7: X-ray reflectometry measurement details and Supplementary Figure S11).

\section{CONCLUSIONS}

Synthesis of NC-based membranes directly grown on porous polymeric substrates was achieved by the use of a simple, environmentally friendly and scalable process which involves the use of a PVP sacrificial layer coating followed by a nanostructured carbon film deposition by plasma-assisted sputtering (HiPIMS). Detailed characterization revealed a nanostructure dependency on the sputter gases mixtures during the deposition of carbon membranes, in which nitrogen groups in $s p^{2}$ carbon networks seem to be a key factor for good rejection and water permeation characteristics. From cross-flow evaluations, the NC-based membranes produced using Ar: $\mathrm{N}_{2}: \mathrm{CH}_{4}$ sputter mixture are the ones showing the best results, with salt rejection rates above $96 \%$ and permeances ca. $22.91 \mathrm{~m}^{-2} \mathrm{~h}^{-1} \mathrm{MPa}^{-1}$ (for $0.2 \mathrm{wt} \% \mathrm{NaCl}$ water), outperforming previous attempts for producing carbon-based membranes for water desalination applications. Results from chlorine-induced degradation evaluations showed that NC-based membranes with high nitrogen content are particularly weak against chlorine attack, but with increasing resistance when nitrogen concentration decreases, with a threshold around $16 \mathrm{vol} \%$. This nitrogen concentration produces membranes with high resistance to chlorine attack, in terms of reduced degradation of salt rejection, and a substantial increase in permeate water flux.

Simplified theoretical analysis of a-C:N models also revealed structural changes which could be correlated with experimental observations on NC-based membranes. The presence of nitrogen within a-C structures had a direct effect in clustering and thus in space distribution, which combined with the induced polarization of $\mathrm{N}$ groups resulted in increased water permeation capabilities and salt rejection performances.

The NC-based membranes presented here exhibited attractive characteristics for water purification and desalination systems, with potential properties which would contribute to overcome some of the drawbacks of current polymeric based RO technology. Further experimental and theoretical analysis on these carbon nanostructured films are underway, in order to fully develop and establish potential new technologies, which can contribute to solve the increasing necessity of pure water sources.

\section{CONFLICT OF INTEREST}

The authors declare no conflict of interest.

\section{ACKNOWLEDGEMENTS}

This research is supported by the Center of Innovation Program, Global Aqua Innovation Center for Improving Living Standard and Water Sustainability from Japan Science and Technology Agency (JST). J.O.-M. acknowledges the supplementary support from CONACYT, México (Estancias Posdoctorales en el Extranjero, exp. 237337). T.A. and M.E. acknowledge the Earth Simulator Center, in the Japan Marine Science and Technology Center (JAMSTEC) for providing computational resources.

1 Vetter, J. 60 years of DLC coatings: historical highlights and technical review of cathodic arc processes to synthesize various DLC types, and their evolution for industrial applications. Surf. Coat. Technol. 257, 213-240 (2014).

2 Ronkainen, H., Varjus, S., Koskinen, J. \& Holmberg, K. Differentiating the tribological performance of hydrogenated and hydrogen-free DLC coatings. Wear 249, 260-266 (2001).

3 Ahmed, M. H., Byrne, J. A. \& McLaughlin, J. Kinetics and thermodynamics of human serum albumin adsorption on silicon doped diamond like carbon. Mater. Chem. Phys. 154, 84-93 (2015).

4 Cui, J., Qiang, L., Zhang, B., Yang, T. \& Zhang, J. Lower friction and higher wear resistance of fluorine-incorporated amorphous carbon films. Surf. Interface Anal. 45, 1329-1333 (2013).

5 Dwivedi, N., Kumar, S., Singh, S. \& Malik, H. K. Oxygen modified diamond-like carbon as window layer for amorphous silicon solar cells. Sol. Energy 86, 220-230 (2012).

6 Liao, W.-H., Lin, C.-R., Wei, D.-H., Shen, Y.-R., Li, Y.-C., Lee, J.-A. \& Liang, C.-Y. Concurrent improvement in biocompatibility and bioinertness of diamond-like carbon films with nitrogen doping. J. Biomed. Mater. Res. A 100, 3151-3156 (2012).

7 Shirakura, A., Nakaya, M., Koga, Y., Kodama, H., Hasebe, T. \& Suzuki, T. Diamond-like carbon films for PET bottles and medical applications. Thin Solid Films 494, 84-91 (2006).

8 Boutroy, N., Pernel, Y., Rius, J. M., Auger, F., von Bardeleben, H. J., Cantin, J. L. Abel, F., Zeinert, A., Casiraghi, C., Ferrari, A. C. \& Robertson, J. Hydrogenated amorphous carbon film coating of PET bottles for gas diffusion barriers. Diam. Relat. Mater. 15, 921-927 (2006).

9 Karan, S., Samitsu, S., Peng, X., Kurashima, K. \& Ichinose, I. Ultrafast viscous permeation of organic solvents through diamond-like carbon nanosheets. Science $\mathbf{3 3 5}$ 444-447 (2012).

10 Vergöhl, M., Bruns, S., Rademacher, D. \& Bräuer, G. Industrial-scale deposition of highly uniform and precise optical interference filters by the use of an improved cylindrical magnetron sputtering system. Surf. Coat. Technol. 267, 53-58 (2015).

11 Matias, V. \& Hammond, R. H. Ion beam induced crystalline texturing during thin film deposition. Surf. Coat. Technol. 264, 1-8 (2015)

12 Ismail, R. A., Hamoudi, W. K. \& Saleh, K. K. Effect of rapid thermal annealing on the characteristics of amorphous carbon/n-type crystalline silicon heterojunction solar cells. Mater. Sci. Semicond. Process 21, 194-199 (2014).

13 da Silva, D. S., Côrtes, A. D. S., Oliveira, M. H., Motta, E. F., Viana, G. A., Mei, P. R. \& Marques, F. C. Application of amorphous carbon based materials as antireflective coatings on crystalline silicon solar cells. J. Appl. Phys. 110, 043510 (2011).

14 Anders, A. A review comparing cathodic arcs and high power impulse magnetron sputtering (HiPIMS). Surf. Coat. Technol. 257, 308-325 (2014).

15 Hinman, P. V., Bell, A. T. \& Shen, M. Composite reverse osmosis membranes prepared by plasma polymerization of allylamine. Evaluation of membrane performance for the treatment of washwater and its components. J. Appl. Polym. Sci. 23, 3651-3656 (1979).

16 Yasuda, H. \& Lamaze, C. E. Preparation of reverse osmosis membranes by plasma polymerization of organic compounds. J. Appl. Polym. Sci. 17, 201-222 (1973).

17 Peric, D., Bell, A. T. \& Shen, M. Reverse osmosis characteristics of composite membranes prepared by plasma polymerization of allylamine. Effects of deposition conditions. J. Appl. Polym. Sci. 21, 2661-2673 (1977).

$18 \mathrm{Li}, \mathrm{A}$., Mu, Y., Jiang, W. \& Wan, X. A mussel-inspired adhesive with stronger bonding strength under underwater conditions than under dry conditions. Chem. Commun. 51, 9117-9120 (2015).

19 Nakahigashi, T., Tanaka, Y., Miyake, K. \& Oohara, H. Properties of flexible DLC film deposited by amplitude-modulated RF P-CVD. Tribol. Int. 37, 907-912 (2004).

20 Pei, Y. T., Bui, X. L. \& De Hosson, J. T. M. Flexible protective diamond-like carbon film on rubber. Scr. Mater. 63, 649-652 (2010). 
21 Bhattacharyya, S., Lübbe, M., Bressler, P., Zahn, D. R. \& Richter, F. Structure of nitrogenated amorphous carbon films from NEXAFS. Diam. Relat. Mater. 11, 8-15 (2002).

22 Titantah, J. T. \& Lamoen, D. Carbon and nitrogen 1s energy levels in amorphous carbon nitride systems: XPS interpretation using first-principles. Diam. Relat. Mater. 16, 581-588 (2007).

23 Gammon, W. J., Kraft, O., Reilly, A. C. \& Holloway, B. C. Experimental comparison of N (1s) X-ray photoelectron spectroscopy binding energies of hard and elastic amorphous carbon nitride films with reference organic compounds. Carbon 41, 1917-1923 (2003).

24 Truica-Marasescu, F. \& Wertheimer, M. R. Nitrogen-rich plasma-polymer films for biomedical applications. Plasma Process. Polym. 5, $44-57$ (2008).

25 Ferrari, A. C. \& Robertson, J. Raman spectroscopy of amorphous, nanostructured, diamond-like carbon, and nanodiamond. Philos. Trans. A. Math. Phys. Eng. Sci. 362, 2477-2512 (2004).

26 Robertson, J. Diamond-like amorphous carbon. Mater. Sci. Eng. R Rep. 37, 129-281 (2002).

27 Durand-Drouhin, O., Benlahsen, M., Clin, M. \& Bouzerar, R. Deposition mechanism of sputtered amorphous carbon nitride thin film. Appl. Surf. Sci. 223, 269-274 (2004).

28 Othman, M., Ritikos, R., Khanis, N. H., Rashid, N. M. A., Gani, S. M. A. \& Rahman, S. A. Effects of hydrogen dilution on CNx film properties deposited using if PECVD from a mixture of ethane, nitrogen and hydrogen. Mater. Chem. Phys. 144, 377-384 (2014).
29 Lee, K. P., Arnot, T. C. \& Mattia, D. A review of reverse osmosis membrane materials for desalination-development to date and future potential. J. Memb. Sci. 370, 1-22 (2011).

30 Ichinose, I., Peng, X., Samitsu, S. \& Karan, S. Method for Producing Nanocarbon Film. Japan, Patent number 2012-036061; filed 11 August 2010, and issued 23 February 2012.

31 Merlet, C., Rotenberg, B., Madden, P. A., Taberna, P.-L., Simon, P., Gogotsi, Y. \& Salanne, M. On the molecular origin of supercapacitance in nanoporous carbon electrodes. Nat. Mater. 11, 306-310 (2012).

(c) (i) This work is licensed under a Creative Commons Attribution 4.0 International License. The images or other third party material in this article are included in the article's Creative Commons license, unless indicated otherwise in the credit line; if the material is not included under the Creative Commons license, users will need to obtain permission from the license holder to reproduce the material. To view a copy of this license, visit http:// creativecommons.org/licenses/by/4.0/

Supplementary Information accompanies the paper on the NPG Asia Materials website (http://www.nature.com/am) 